\title{
Small is beautiful: diversity of freshwater ostracods (Crustacea, Ostracoda) in marginal habitats of the province of Parma (Northern Italy)
}

\author{
Giampaolo ROSSETTI*, Koen MARTENS ${ }^{1)}$, Claude MEISCH ${ }^{2)}$, Stefano TAVERNELLI and Valentina PIERI \\ Department of Environmental Sciences, University of Parma, Viale G.P. Usberti 33A, 43100 Parma, Italy \\ ${ }^{1)}$ Royal Belgian Institute of Natural Sciences, Freshwater Biology, Vautierstraat 29, 1000 Brussels, Belgium \\ ${ }^{2)}$ Musée d'Histoire Naturelle, 25 rue Münster, 2160 Luxembourg, Grand Duchy of Luxembourg \\ *e-mail corresponding author: giampaolo.rossetti@unipr.it
}

\begin{abstract}
A study on the distribution of ostracods in small freshwater habitats of the province of Parma was conducted from May 2004 to December 2005. Vernal pools, ponds, shallow lakes, fountains, springs, ditches, canals, and streams were among the most common types of aquatic systems included in this survey. Altogether, 90 sites located between 24 and $1557 \mathrm{~m}$ a.s.l. were visited, and 125 samples were collected. 41 samples did not contain ostracods, and 7 samples were not considered because only immature specimens or empty valves were found. Consequently, 77 samples from 58 sites were analysed. Whenever possible, ostracod identification was done at the species level based on the morphology of both valves and limbs. Thirty-eight taxa from 20 genera belonging to the families Candonidae, Ilyocyprididae, Cyprididae, and Notodromadidae were recorded. This roughly accounts for one-third of the estimated number of non-marine ostracod species in Italy. Scanning electron microscopy images of valves are provided. The maximum number of species per site was six. The most frequent species were Heterocypris incongruens, Eucypris virens, Pseudocandona pratensis, Cypria ophthalmica, and Tonnacypris lutaria. Of particular relevance is the occurrence of species with a limited known distribution in Italy, viz. Ilyocypris monstrifica, Potamocypris villosa, Candona cf. lindneri, Fabaeformiscandona breuili and Physocypria kraepelini. A putative new species of the genus Candona, here left in open nomenclature, is also reported. Our results highlight the importance of so-called "marginal" aquatic habitats as potential biodiversity hotspots, in spite of the general lack of interest shown by freshwater ecologists and taxonomists in undertaking scientific investigations in these environments.
\end{abstract}

Key words: inland water bodies, Recent ostracods, taxonomy, biodiversity

\section{INTRODUCTION}

The ostracods (class Ostracoda) are the most diverse group of crustaceans with an estimated range from 4000 to 30000 living species (Meisch 2000). Their most salient characteristic is the presence of a bivalve carapace that can completely enclose a laterally-compressed and weakly-segmented body. They can be found in inland and marine waters, in interstitial and groundwater environments and also in semi-terrestrial habitats such as leaf litter. All Recent freshwater ostracods belong to the order Pocodocopida. They mainly colonize benthic and periphytic habitats and feed on both living and detrital particles; more rarely, they can be predators or parasites.

This research aims to provide a faunal account of ostracods living in freshwater habitats of the province of Parma (Northern Italy) and investigate their distributional patterns. A previous study (Rossetti et al. 2005) examined the composition of the ostracod communities in 20 lowland springs within the province. The considered area is rich in waters, both lentic and lotic. It is characterized by a strong south-north altitudinal gradient from the lowlands to the Apennine ridge (with a maximum elevation of $1861 \mathrm{~m}$ a.s.l.) and by a variety of climatic, geological and land-use conditions.
We primarily focussed our attention on the prevalent types of aquatic habitats in this territory, i.e. small water bodies, either natural or artificial. The marginality of these ecosystems is generally related to their isolation, unpredictable duration, natural or anthropic disturbance and, in many cases, deficient regulatory and legislative policies for their protection. This results in biological communities that are mainly composed of species able to cope with harsh environmental conditions, for example, a high variability of hydrological and hydrochemical characteristics. This study was stimulated by preliminary observations indicating an unexpectedly diverse ostracofauna in minor aquatic ecosystems and seeks, along with data recently collected from other parts of the country (e.g., Rossetti et al. 2004; Pieri et al. 2006a, 2006b), to contribute to the revision and updating of the checklist of Italian ostracods (Ghetti \& McKenzie 1981).

\section{MATERIALS AND METHODS}

Sampling was performed from May 2004 to December 2005 in 90 sites located between 24 and $1557 \mathrm{~m}$ a.s.l. Sampling locations were chosen to possibly encompass the most common types of marginal aquatic habitats and to cover the entire altitudinal range of the 

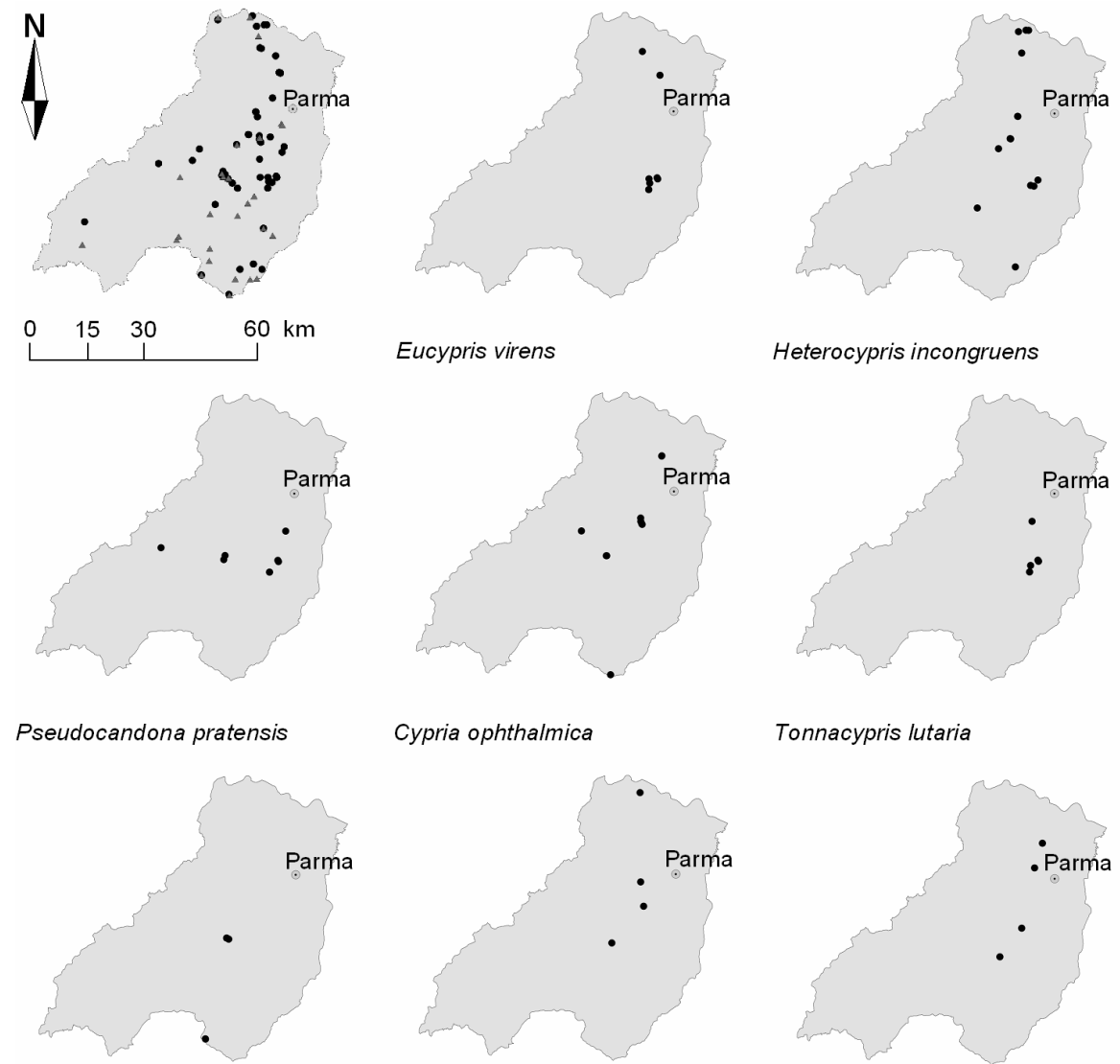

Tonnacypris lutaria

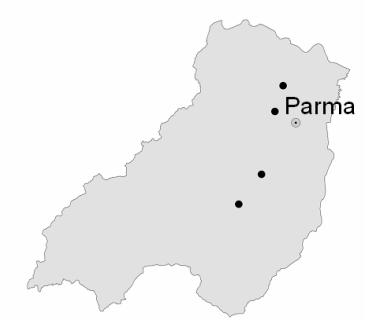

Prionocypris zenkeri

Fig. 1. Map of the province of Parma showing the sampling sites considered in this study (circles) and those not included (triangles) because no ostracods were found or only immature stages or empty valves were present (upper left corner), and distribution of the most frequently found ostracod species.

province of Parma. Qualitative ostracod samples were collected using a $250 \mu \mathrm{m}$ hand net or, when not possible due to the limited size of the sampled site, with cloth coffee filters. In total, 125 samples were collected and transferred to the laboratory, where living specimens were picked up under a binocular microscope within 24 hours of sampling and then fixed in $80 \%$ ethanol. In 13 sites, ostracods were gathered on different dates. Fortyone samples did not contain ostracods, while an additional 7 samples were not considered because only immature specimens or empty valves were found. Therefore, 77 samples from 57 sites were eventually analysed (Tab. 1, Fig. 1). The specific allocation of the collected material was based on adult specimens; in a few cases (Candona sp. B in S72, Candona sp. C in S24, and Pseudocandona sp. in S57 and S72) the identification remained at the generic level because only a few individuals, damaged material or females were available for the taxonomic analysis. Both soft parts (dissected in glycerine and stored in sealed slides) and valves (stored dry in micropaleontological slides and used for scanning microphotographs) were checked for species identification, using Fox (1965) for Chlamydotheca incisa, González-Mozo et al. (1996) for the genus
Herpetocypris, and Meisch (2000) for the remaining taxa. The analyzed material is deposited at the Department of Environmental Sciences, University of Parma (preceded by the code "VP") and in the Ostracod Collection of the Royal Belgian Institute of Natural Sciences, Brussels (preceded by the codes "OC" and "RP"). We used the following abbreviations in our figures: L: left; R: right; V: valve; Cp: carapace; dv: dorsal view; vv: ventral view; lv: lateral view; iv: internal view; ev: external view.

\section{RESULTS}

Altogether, 38 taxa in 20 genera belonging to 4 families (Candonidae, Ilyocyprididae, Cyprididae, and Notodromadidae) were identified (Tab. 2; Figs 2-5). The maximum number of species reported in a single sample (S58) was 6, namely Chlamydotheca incisa, Heterocypris incongruens, $H$. salina, Dolerocypris sinensis, Trajancypris clavata and Ilyocypris monstrifica. Other samples with a relatively high number of species were S57 and S67, both containing 4 species. On the other hand, 16 taxa were found only in one site (Tab. 2). 
Tab. 1. Geographic characteristics of sampling stations and list of samples used in this study.

\begin{tabular}{|c|c|c|c|c|c|c|}
\hline Site name & Coordinates & $\begin{array}{l}\text { Height } \\
\text { (m a.s.1.) }\end{array}$ & Habitat type & Municipality & Sampling date & $\begin{array}{l}\text { Sample } \\
\text { code }\end{array}$ \\
\hline Montevacà & 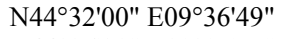 & 777 & Fountain basin & Bedonia & 13 Mar 2005 & S01 \\
\hline Calestano & N4436'39" E1007'55" & 391 & Fountain basin & Calestano & 16 Jun 2005 & S02 \\
\hline $\mathrm{SP} 15 \mathrm{~km} 12.5$ & $\mathrm{~N} 44^{\circ} 34^{\prime} 20^{\prime \prime} \mathrm{E} 10^{\circ} 03^{\prime} 20^{\prime \prime}$ & 721 & Fountain basin & Calestano & 19 Sep 2004 & S03 \\
\hline Canale del Mulino & N44ㄴ4'23" E10¹0'20" & 107 & Canal & Collecchio & 05 May 2004 & S04 \\
\hline Case Folli & N44ㄴ4'00" E10¹4'43" & 129 & Ditch & Collecchio & 16 Jun 2005 & S05 \\
\hline Corte Giarola & N44ㄴ4'25" E10¹0'14" & 107 & Temporary pool & Collecchio & 05 May 2004 & S06 \\
\hline Lago Chiesuole & 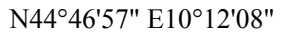 & 73 & Artificial lake & Collecchio & 05 May 2004 & S07 \\
\hline Qualatico & $\mathrm{N} 44^{\circ} 42^{\prime} 58^{\prime \prime} \mathrm{E} 10^{\circ} 07^{\prime} 50^{\prime \prime}$ & 117 & Temporary pool & Collecchio & 05 May 2004 & S08 \\
\hline Rio del Lago Santo & $\mathrm{N} 44^{\circ} 24^{\prime} 05^{\prime \prime} \mathrm{E} 10^{\circ} 00^{\prime} 22^{\prime \prime}$ & 1515 & Creek & Corniglio & 27 Jul 2005 & S09 \\
\hline Fontana del Lago Santo & N44ํ2'07" E1000'21" & 1510 & Fountain basin & Corniglio & 27 Jul 2005 & S10 \\
\hline Ponte del Cogno & 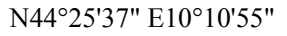 & 986 & Fountain basin & Corniglio & $27 \mathrm{Jul} 2005$ & $\mathrm{~S} 11$ \\
\hline Via Bambozza & N444ㄴ'47" E10¹7'05" & 153 & Ditch & Felino & 16 Jun 2005 & $\mathrm{~S} 12$ \\
\hline Fonte Lupo - Rio Lusore & N4439'07" E1005'00" & 480 & Rheocrenic spring & Fornovo di Taro & $\begin{array}{l}27 \text { Apr } 2005 \\
17 \text { Nov } 2005\end{array}$ & $\begin{array}{l}\text { S13 } \\
\text { S14 }\end{array}$ \\
\hline Laghetto di Villanova & 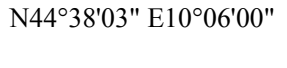 & 316 & Permanent pond & Fornovo di Taro & $\begin{array}{l}04 \text { May } 2005 \\
22 \text { Nov } 2005\end{array}$ & $\begin{array}{l}\text { S15 } \\
\text { S16 }\end{array}$ \\
\hline Prima Bratte & N443'ㄹ' $22^{\prime \prime}$ E1005'09" & 573 & Rheocrenic spring & Fornovo di Taro & $\begin{array}{l}04 \text { May } 2005 \\
17 \text { Nov } 2005\end{array}$ & $\begin{array}{l}\text { S17 } \\
\text { S18 }\end{array}$ \\
\hline Seconda Bratte & N44웅' $28^{\prime \prime} 10^{\circ} 05^{\prime} 17^{\prime \prime}$ & 545 & Rheocrenic spring & Fornovo di Taro & 17 Nov 2005 & S19 \\
\hline Rio delle Bighe & N443ㅇ'53" E1004'57" & 490 & Streamlet & Fornovo di Taro & $\begin{array}{l}24 \text { Mar } 2005 \\
04 \text { May } 2005 \\
17 \text { Nov } 2005\end{array}$ & $\begin{array}{l}\text { S20 } \\
\text { S21 } \\
\text { S22 }\end{array}$ \\
\hline Rio di Chiastra & 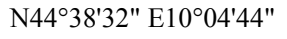 & 563 & Streamlet & Fornovo di Taro & 22 Nov 2005 & $\mathrm{~S} 23$ \\
\hline Fontana Sgalara & $\mathrm{N} 44^{\circ} 38^{\prime} 44^{\prime \prime} \mathrm{E} 10^{\circ} 05^{\prime} 20^{\prime \prime}$ & 523 & Fountain basin & Fornovo di Taro & 17 Nov 2005 & $\mathrm{~S} 24$ \\
\hline Sorgente Sgalara & 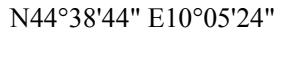 & 506 & Rheocrenic spring & Fornovo di Taro & $\begin{array}{l}10 \text { May } 2005 \\
17 \text { Nov } 2005\end{array}$ & $\begin{array}{l}\text { S25 } \\
\text { S26 }\end{array}$ \\
\hline Case Manfredelli & 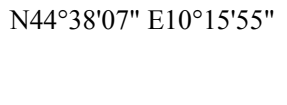 & 431 & Temporary pool & Langhirano & $\begin{array}{l}19 \text { Mar } 2005 \\
22 \text { Apr } 2005 \\
20 \text { Oct } 2005\end{array}$ & $\begin{array}{l}\text { S27 } \\
\text { S28 } \\
\text { S29 }\end{array}$ \\
\hline Costa di Castrignano P1 & 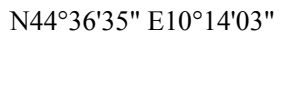 & 469 & Temporary pool & Langhirano & $\begin{array}{l}18 \text { Mar } 2005 \\
22 \text { Apr } 2005 \\
20 \text { Oct } 2005\end{array}$ & $\begin{array}{l}\text { S30 } \\
\text { S31 } \\
\text { S32 }\end{array}$ \\
\hline Costa di Castrignano P2 & N44웅' $35^{\prime \prime}$ E10¹4'03" & 469 & Temporary pool & Langhirano & $\begin{array}{l}18 \text { Mar } 2005 \\
22 \text { Apr } 2005 \\
20 \text { Oct } 2005\end{array}$ & $\begin{array}{l}\text { S33 } \\
\text { S34 } \\
\text { S35 }\end{array}$ \\
\hline Mattaleto & N443'ㄱ' $25^{\prime \prime}$ E10¹4'57" & 385 & Temporary pool & Langhirano & 22 Apr 2005 & $\mathrm{~S} 36$ \\
\hline Strognano & 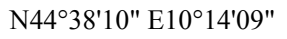 & 436 & Temporary pool & Langhirano & 22 Apr 2005 & S37 \\
\hline Tordenaso & 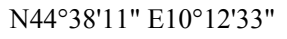 & 431 & Temporary pool & Langhirano & 22 Apr 2005 & $\mathrm{~S} 38$ \\
\hline Vallo di Sotto & N443'ㄱ' $31^{\prime \prime}$ E1014'17" & 410 & Temporary pool & Langhirano & $\begin{array}{l}18 \text { Mar } 2005 \\
22 \text { Apr } 2005 \\
10 \text { Jun } 2005\end{array}$ & $\begin{array}{l}\text { S39 } \\
\text { S40 } \\
\text { S41 }\end{array}$ \\
\hline Vidiana P1 & 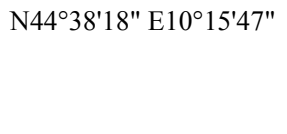 & 279 & Temporary pool & Langhirano & $\begin{array}{l}18 \text { Mar } 2005 \\
22 \text { Apr } 2005 \\
10 \text { Jun } 2005 \\
20 \text { Oct } 2005\end{array}$ & $\begin{array}{l}\text { S42 } \\
\text { S43 } \\
\text { S44 } \\
\text { S45 }\end{array}$ \\
\hline Capanna Biancani & $\mathrm{N} 44^{\circ} 21^{\prime} 16^{\prime \prime} \mathrm{E} 10^{\circ} 05^{\prime} 52^{\prime \prime}$ & 1557 & Bog & Monchio delle Corti & 04 Aug 2005 & S46 \\
\hline Ponte di Lugagnano & N442ㄴ'52" E1008'10" & 680 & Fountain basin & Monchio delle Corti & $\begin{array}{l}\text { 16 Jun } 2004 \\
04 \text { Aug } 2005\end{array}$ & $\begin{array}{l}\text { S47 } \\
\text { S48 }\end{array}$ \\
\hline La Casella P1 & $\mathrm{N} 44^{\circ} 47^{\prime} 40^{\prime \prime} \mathrm{E} 10^{\circ} 11^{\prime} 53^{\prime \prime}$ & 67 & Temporary pool & Noceto & 05 May 2004 & S49 \\
\hline La Casella P2 & $\mathrm{N} 44^{\circ} 47^{\prime} 41^{\prime \prime} \mathrm{E} 10^{\circ} 11^{\prime} 50^{\prime \prime}$ & 67 & Temporary pool & Noceto & 05 May 2004 & $\mathrm{~S} 50$ \\
\hline Vairo Inferiore & 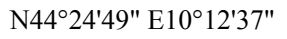 & 731 & Fountain basin & Palanzano & 04 Aug 2005 & S51 \\
\hline Strada Mulattiera & N4449'39" E10¹5'18" & 44 & Ditch & Parma & 15 Jun 2005 & S52 \\
\hline Torrazza & N444'ㄱ' $33^{\prime \prime}$ E10¹7'29" & 139 & Ditch & Parma & 16 Jun 2005 & S53 \\
\hline Ongina Morta & N4501'06" E1004'21" & 33 & Dead river channel & Polesine Parmense & 03 May 2004 & S54 \\
\hline Cava dei Francesi 2 & N4501'36" E10¹1'27" & 25 & Artificial lake & Roccabianca & 08 Jun 2005 & S55 \\
\hline Fossa 1 & N45ํ0' $16^{\prime \prime} \mathrm{E} 10^{\circ} 13^{\prime} 48^{\prime \prime}$ & 27 & Ditch & Roccabianca & 15 Jun 2005 & S56 \\
\hline Fossa 2 & $\mathrm{~N} 45^{\circ} 00^{\prime} 15^{\prime \prime} \mathrm{E} 10^{\circ} 14^{\prime} 20^{\prime \prime}$ & 26 & Ditch & Roccabianca & 15 Jun 2005 & S57 \\
\hline Rigosa Vecchia & 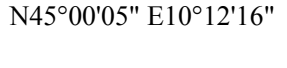 & 27 & Canal & Roccabianca & $\begin{array}{l}17 \text { May } 2004 \\
15 \text { Jun } 2005\end{array}$ & $\begin{array}{l}\text { S58 } \\
\text { S59 }\end{array}$ \\
\hline Bosco della Capannella & N444' $11^{\prime \prime}$ E10¹2'29" & 164 & Temporary pool & Sala Baganza & 02 Dec 2004 & S60 \\
\hline Castellaro & N444ㅇ'50" E10¹2'29" & 213 & Isolated river pool & Sala Baganza & 16 Jun 2005 & S61 \\
\hline Lago Antonia & 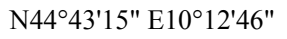 & 191 & Permanent pond & Sala Baganza & 02 Dec 2004 & S62 \\
\hline Lago di Monte Tinto & N44ㄴ3'41" E10¹2'29" & 179 & Permanent pond & Sala Baganza & 02 Dec 2004 & S63 \\
\hline Lago di Ponte Verde & N444'ㄹ' $22^{\prime \prime}$ E10¹2'39" & 193 & Artificial lake & Sala Baganza & 02 Dec 2004 & S64 \\
\hline
\end{tabular}


Tab. 1. Continuation

\begin{tabular}{|c|c|c|c|c|c|c|}
\hline Site name & Coordinates & $\begin{array}{l}\text { Height } \\
\text { (m a.s.1.) }\end{array}$ & Habitat type & Municipality & Sampling date & $\begin{array}{l}\text { Sample } \\
\text { code }\end{array}$ \\
\hline Case Ziliotti & 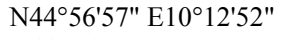 & 30 & Ditch & San Secondo P.se & 21 Jan 2005 & S65 \\
\hline Colmignola & 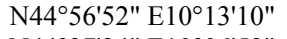 & 30 & Ditch & San Secondo P.se & 03 May 2004 & S66 \\
\hline Lesignano Palmia & N443'ㄴ' $24^{\prime \prime}$ E1006'52" & 370 & Fountain basin & Terenzo & 26 May 2005 & S67 \\
\hline Carpaneto & 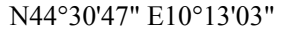 & 541 & Fountain basin & Tizzano Val Parma & 02 Jul 2004 & S68 \\
\hline Canale Lazzaretto & 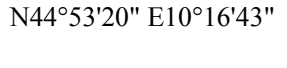 & 24 & Ditch & Trecasali & $\begin{array}{l}\text { 03 May } 2004 \\
21 \text { Jan } 2005\end{array}$ & $\begin{array}{l}\text { S69 } \\
\text { S70 }\end{array}$ \\
\hline Canale Lorno & 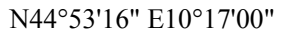 & 27 & Canal & Trecasali & 15 Jun 2005 & S71 \\
\hline Cimitero di Trecasali & $\mathrm{N} 45^{\circ} 55^{\prime} 48^{\prime \prime} \mathrm{E} 10^{\circ} 16^{\prime} 05^{\prime \prime}$ & 28 & Ditch & Trecasali & 15 Jun 2005 & S72 \\
\hline La Commenda & N445'14" E1016'59" & 28 & Limnocrenic spring & Trecasali & 15 Jun 2005 & S73 \\
\hline Lazzaretto 2 & N445'ㄴ' $17^{\prime \prime}$ E10¹6'58" & 24 & Ditch & Trecasali & 15 Jun 2005 & S74 \\
\hline Bivio Aie - Sitla & N444ㅇ'45" E0958'50" & 377 & Stillicide on mosses & Varano de' Melegari & 24 Jul 2005 & S75 \\
\hline Riviano Chiesa & N444ㄴ'24" E1000'17" & 423 & Fountain basin & Varano de' Melegari & 20 Nov 2005 & S76 \\
\hline Case Buffalora & N444ㅇ'22" E0951'56" & 374 & Temporary pool & Varsi & 13 Mar 2005 & S77 \\
\hline
\end{tabular}

Tab. 2. Taxonomic list of ostracods found in this study, and stations (codes as in Tab. 1) where they were collected.

Superfamily Cypridoidea s. str. Baird, 1845

Family Candonidae Kaufmann, 1900

Subfamily Candoninae Kaufmann, 1900

Genus Candona s. str. Baird, 1845

Candona cf. lindneri Petkovski, $1969 \quad$ S63, S70

Candona neglecta Sars, $1887 \quad$ S19, S25, S26

Candona sp. A $\quad$ S34, S35

Candona sp. B $\quad \mathrm{S} 72$

$\begin{array}{ll}\text { Candona sp. C } & \text { S24 }\end{array}$

Genus Fabaeformiscandona Krstić, 1972

Fabaeformiscandona breuili (Paris ,1920) nov. comb. S13

Genus Pseudocandona Kaufmann, 1900

Pseudocandona lobipes (Hartwig, 1900)

Pseudocandona pratensis (Hartwig, 1901)

Pseudocandona albicans (Brady, 1864)

Pseudocandona sp.

Genus Candonopsis Vávra, 1891 Candonopsis kingsleii (Brady \& Robertson, 1870) S57

Subfamily Cyclocypridinae Kaufmann, 1900

Genus Cypria Zenker, 1854

Cypria ophthalmica (Jurine, 1820)

Genus Physocypria Vávra, 1897

Physocypria kraepelini (G.W. Müller, 1903)

Genus Cyclocypris Brady \& Norman, 1889

Cyclocypris laevis (O.F. Müller, 1776) $\quad$ S73

Cyclocypris ovum (Jurine, 1820) S46

Family Ilyocyprididae Kaufmann, 1900

Subfamily Ilyocypridinae Kaufmann, 1900

Genus Ilyocypris Brady \& Norman, 1889

$\begin{array}{ll}\text { Ilyocypris gibba (Ramdohr, 1808) } & \mathrm{S} 08, \mathrm{~S} 16 \\ \text { Ilyocypris monstrifica (Norman, 1862) } & \mathrm{S} 55, \mathrm{~S} 58 \\ \text { Ilyocypris decipiens } \text { (Masi, 1905) } & \mathrm{S} 16 \\ \text { Ilyocypris bradyi Sars, 1890 } & \mathrm{S} 07, \mathrm{~S} 15, \mathrm{~S} 71 \\ \text { Ilyocypris inermis Kaufmann, 1900 } & \mathrm{S} 18, \mathrm{~S} 38, \mathrm{~S} 74\end{array}$

Family Notodromadidae Kaufmann, 1900

Subfamily Notodromadinae Kaufmann, 1900

Genus Notodromas Lilljeborg, 1853

Notodromas persica Gurney, 1921 
Tab. 2. Continuation

\section{Family Cyprididae Baird, 1845}

Subfamily Eucypridinae Bronshtein, 1947

Genus Eucypris Vávra, 1891

Eucypris virens (Jurine, 1820)

Eucypris cf. pigra (Fischer, 1851)

Genus Prionocypris Brady \& Norman, 1896

Prionocypris zenkeri (Chyzer \& Toth, 1858)

Genus Tonnacypris Diebel \& Pietrzeniuk, 1975

Tonnacypris lutaria (Koch, 1838)

Genus Trajancypris Martens, 1989

Trajancypris clavata (Baird, 1838)

Subfamily Herpetocypridinae Kaufmann, 1900

Genus Herpetocypris Brady \& Norman, 1889

Herpetocypris ef. brevicaudata Kaufmann, 1900

Herpetocypris chevreuxi (Sars, 1896)

Genus Psychrodromus Danielopol \& McKenzie, 1977

Psychrodromus olivaceus (Brady \& Norman, 1889)

Psychrodromus fontinalis (Wolf, 1920)

Subfamily Cypridinae Baird, 1845

Genus Chlamydotheca (Saussure, 1858)

Chlamydotheca incisa (Claus, 1812)

Subfamily Cyprinotinae Bronshtein, 1947

Genus Heterocypris Claus, 1892

Heterocypris incongruens (Ramdohr, 1808)

Heterocypris salina (Brady, 1868)

Heterocypris reptans (Kaufmann, 1900)

Subfamily Dolerocypridinae Triebel, 1961

Genus Dolerocypris Kaufmann, 1900

Dolerocypris sinensis (Sars, 1903)

Subfamily Cypridopsinae Kaufmann, 1900

Genus Cypridopsis Brady, 1867

Cypridopsis vidua (O.F. Müller, 1776)

Genus Potamocypris Brady, 1870

Potamocypris fulva (Brady, 1868)

Potamocypris villosa (Jurine, 1820)
S28, S30, S31, S32, S33, S34, S35, S37, S39, S42, S43, S45, S66, S69, S70

S67

S02, S52, S61, S71

S05, S27, S28, S29, S30, S31, S39, S40, S42

S58

$\mathrm{S} 03, \mathrm{~S} 12$

$\mathrm{S} 64$

S67, S75

$\mathrm{S} 17, \mathrm{~S} 18$

S58, S59

S03, S04, S06, S08, S11, S36, S39, S41, S44, S49, S50, S56, S57, S58, S65

S58

S47, S48, S67, S68

S58

S07, S15, S54, S59, S64

S09, S10, S20, S21, S22, S25, S26

S01, S51, S68 


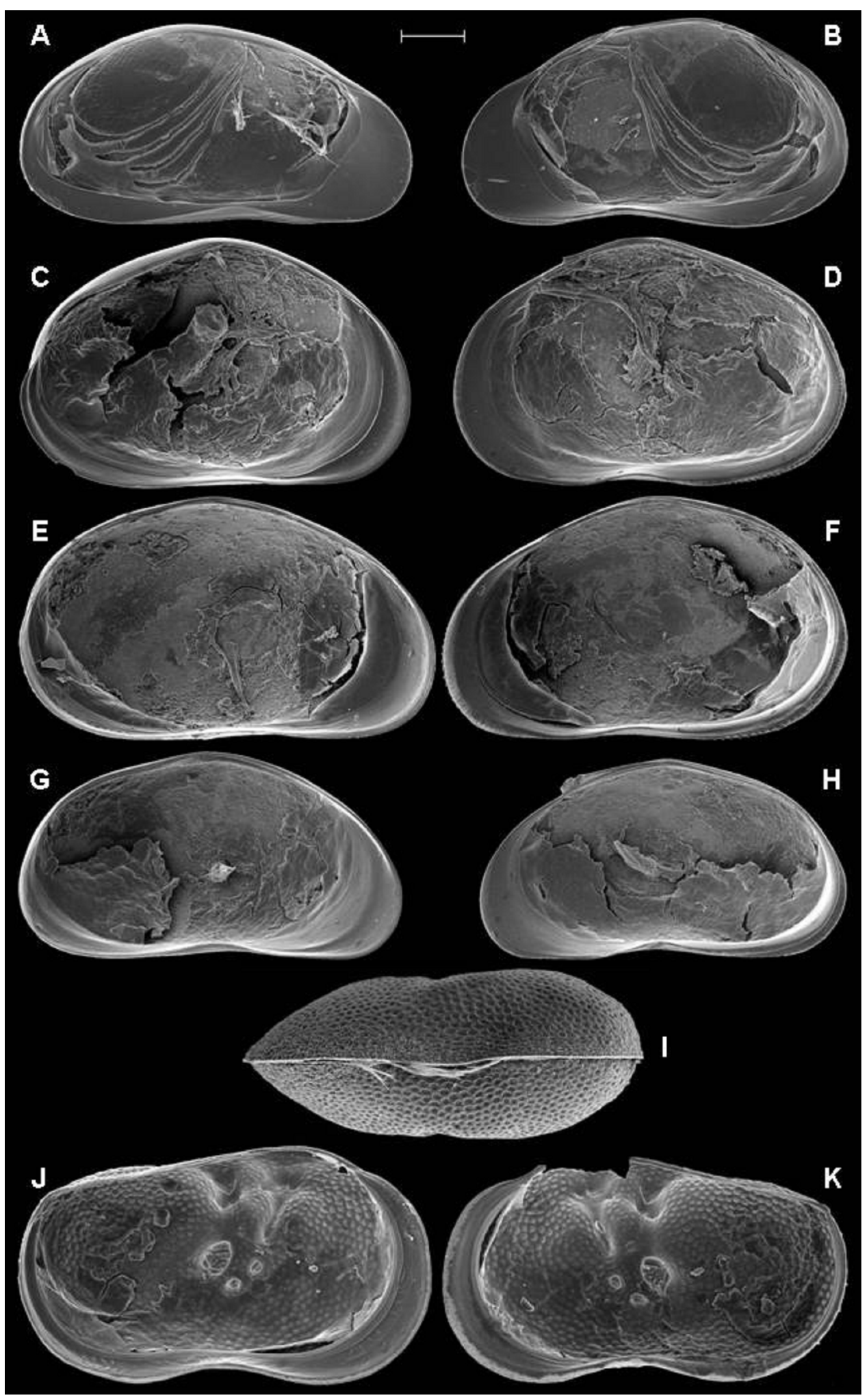

Fig. 2. Candonopsis kingsleii (A-B); Heterocypris salina $(\mathbf{C}-\mathbf{D})$; Heterocypris incongruens $(\mathbf{E}-\mathbf{F})$; Heterocypris reptans $(\mathbf{G}-\mathbf{H})$; Ilyocypris gibba (I-K). All adult specimens. (A): VP0621, male, LV, iv; (B): idem, RV, iv; (C): VP0045, female, LV, iv; (D): idem, RV, iv; (E): VP0226, female, LV, iv; (F): idem, RV, iv; (G): VP0060, female, LV, iv; (H): VP0060, idem, iv; (I): VP0313, Cp, vv; (J): VP0034, female, LV, iv; (K): idem, RV, iv (partially damaged). Scale bar: $167 \mu \mathrm{m}$ for A-B; $200 \mu \mathrm{m}$ for C-H; $130 \mu \mathrm{m}$ for I-K. 


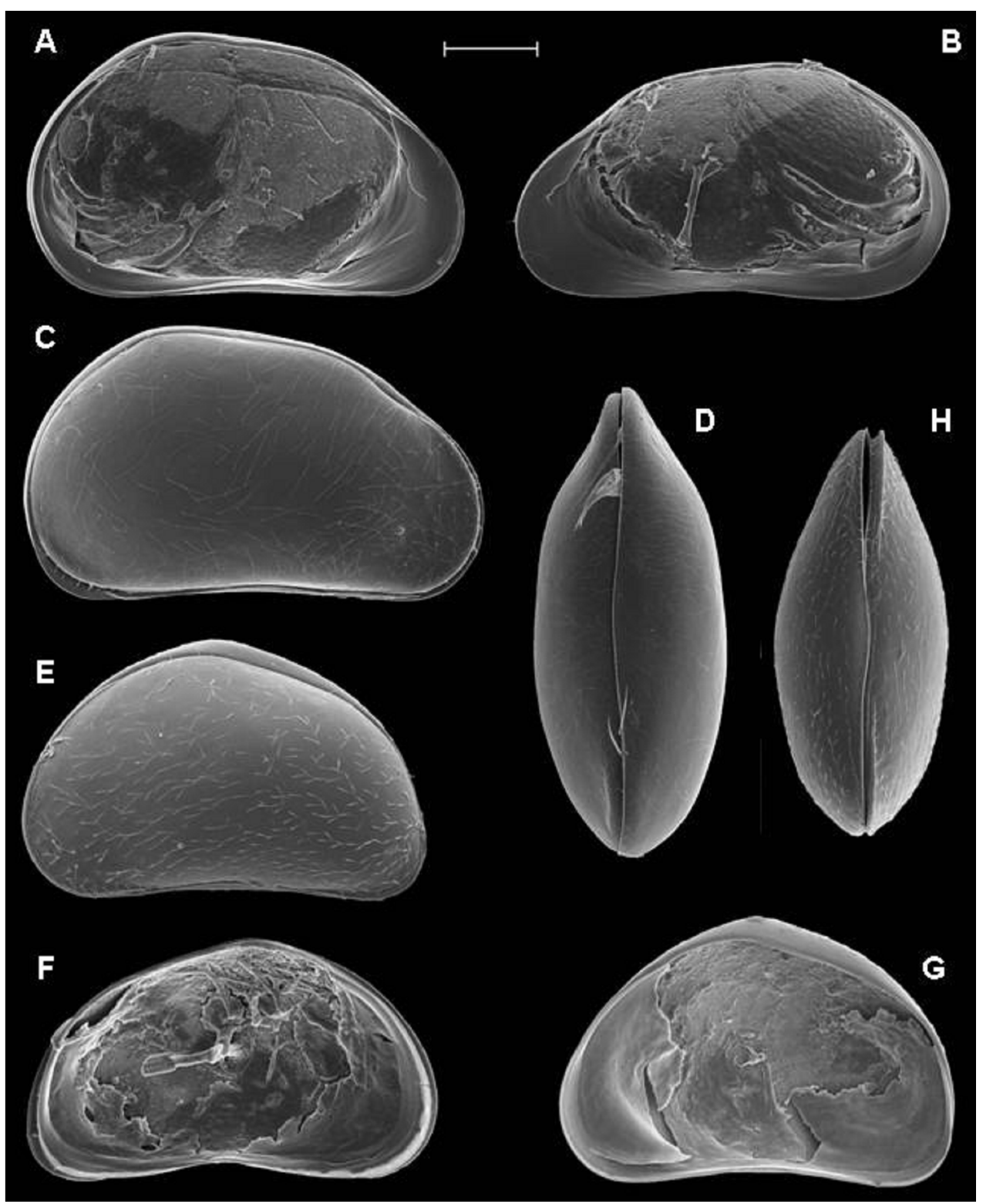

Fig. 3. Pseudocandona pratensis (A-D); Potamocypris villosa $(\mathbf{E}-\mathbf{H})$. All adult specimens. (A): VP0623, male, LV, iv; (B): idem, RV, iv; (C): VP0638, female, Cp, Rlv; (D): VP0639, female, Cp, vv; (E): VP0308, female, Cp, Llv; (F): VP0076, female, LV, iv; (G) idem, RV, iv; VP0307, female, Cp, vv. Scale bar: $200 \mu \mathrm{m}$ for $\mathbf{A}-\mathbf{D}, 160 \mu \mathrm{m}$ for $\mathbf{E}-\mathbf{H}$. 


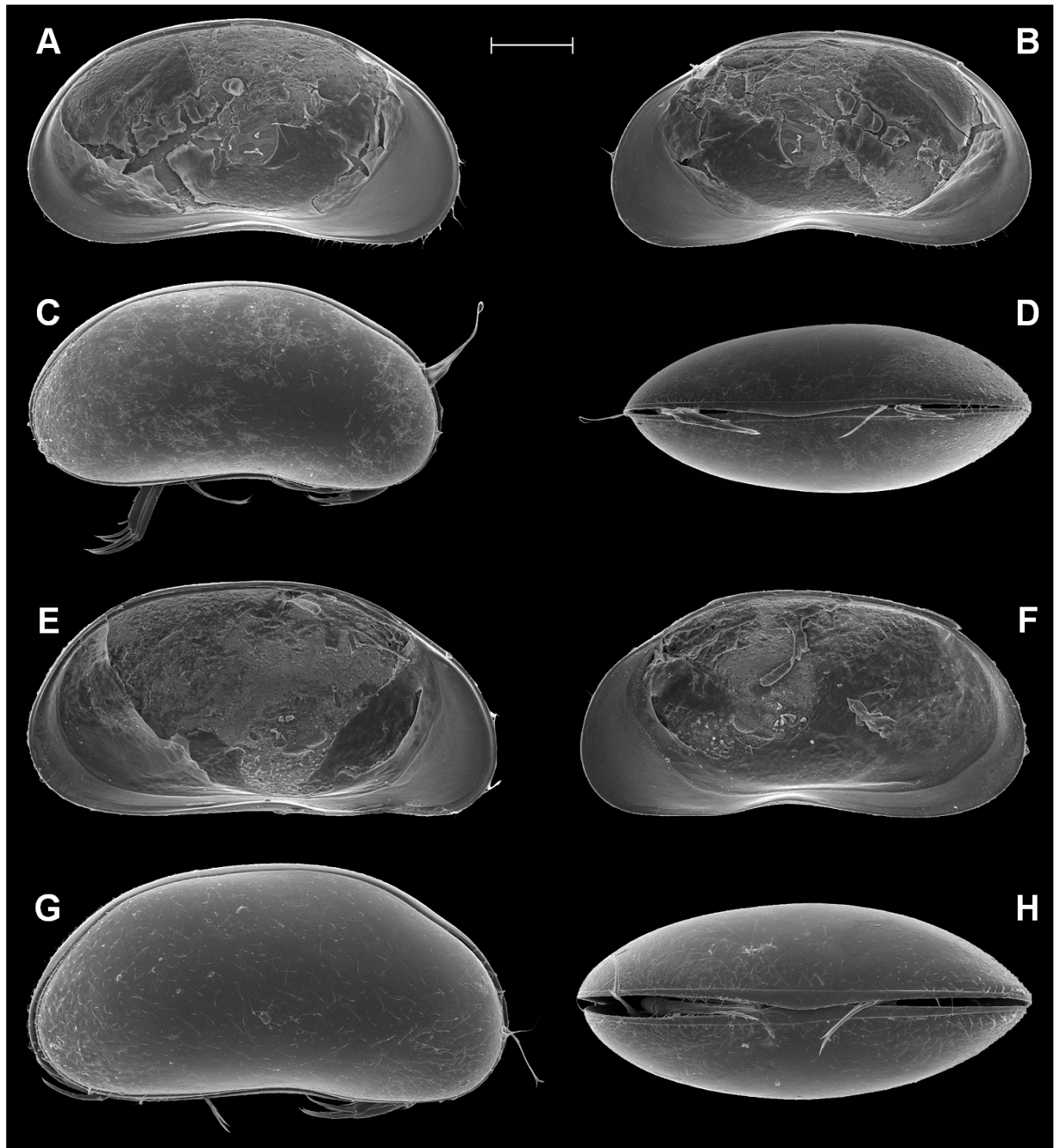

Fig. 4. Psychrodromus fontinalis (A-D); Psychrodromus olivaceus $(\mathbf{E}-\mathbf{H})$. All adult specimens. (A): VP0470, female, LV, iv; (B): idem, RV, iv; (C): VP0645, female, Cp, Rlv; (D): VP0646, female, Cp, vv; (E): VP0622, female, LV, iv; (F): idem, RV, iv; (G): VP0647, female, Cp, Rlv; (H): VP0648, female, Cp, vv. Scale bar: $229 \mu \mathrm{m}$ for A-D; $200 \mu \mathrm{m}$ for E-H. 


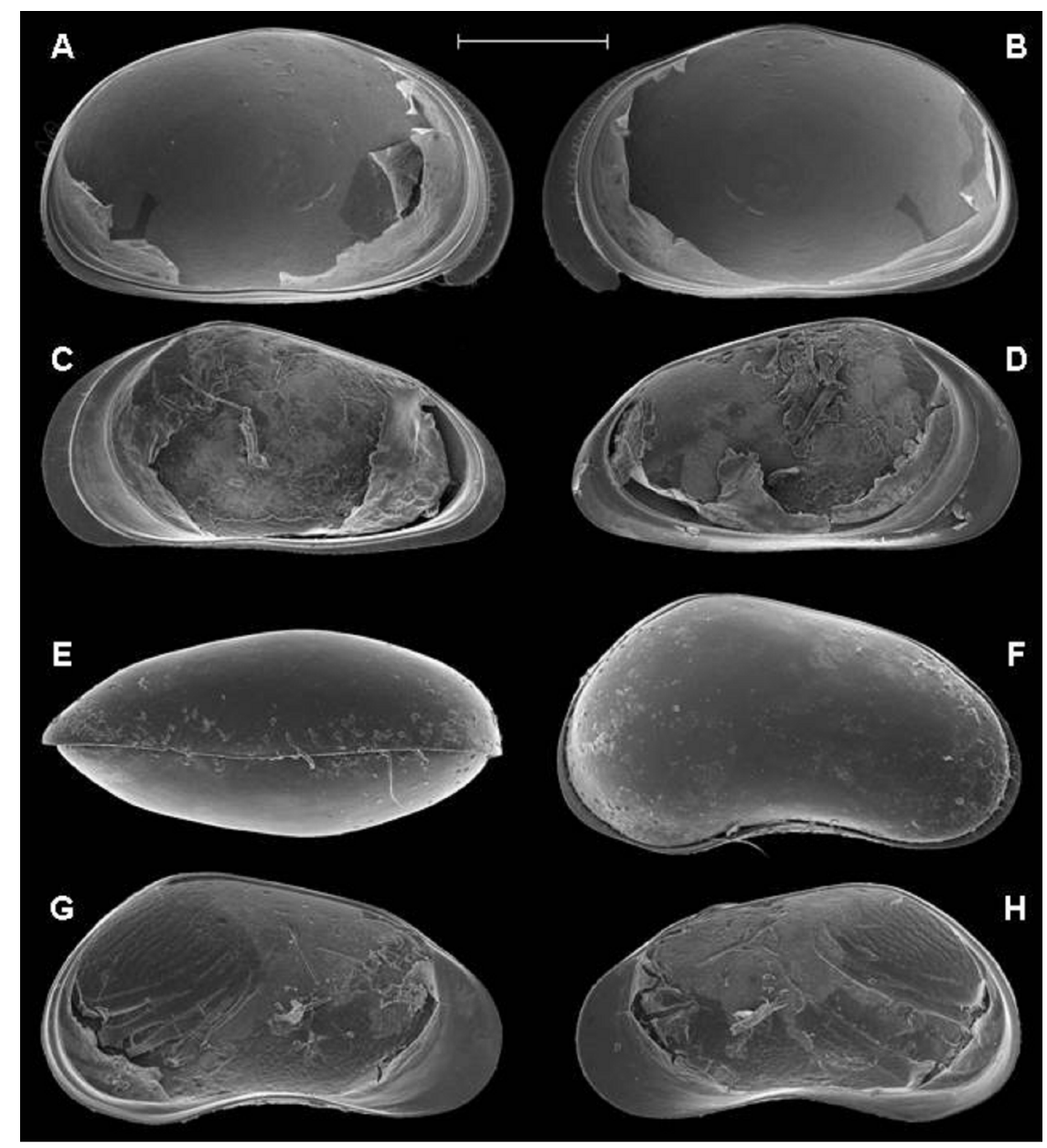

Fig. 5. Chlamydotheca incisa (A-B); Trajancypris clavata (C-D); Candona sp. A (E-H). All adult specimens. (A): RP312, female, LV, iv; (B): idem, RV, iv; (C): VP0027, female, LV, iv; (D): idem, RV, iv; (E):VP0644, female, Cp, vv; (F): VP0643, male, Cp, Rlv; (G): VP0619, male, LV, iv; (H): idem, RV, iv. Scale bar: $800 \mu \mathrm{m}$ for A-B, $750 \mu \mathrm{m}$ for $\mathbf{C}-\mathbf{D}, 500 \mu \mathrm{m}$ for $\mathbf{E}-\mathbf{H}$.

Eucypris virens and Heterocypris incongruens were the most common species, both with 15 records. Eucypris virens was found at altitudes below $500 \mathrm{~m}$ a.s.l., mostly in temporary waters and during winter months. Heterocypris incongruens was reported across a wider range of altitudes (up to $986 \mathrm{~m}$ a.s.1., S11), in different habitat types and over a broader temporal span. Moreover, $H$. incongruens was the only species found in 10 samples out of 15 in contrast to E. virens which was often found with Pseudocandona pratensis (in 6 samples) and Tonnacypris lutaria (in 5 samples). Apart from the above mentioned cases, no other recurrent species associations were observed.

Other species which occurred frequently were Cypria ophthalmica (in 10 samples taken from environments with distinct features), Pseudocandona pratensis and Tonnacypris lutaria (respectively in 11 and 9 samples, predominantly from temporary pools), and Potamocypris fulva (in 7 samples collected from sites characterised by moderate water turbulence) (Tabs 1, 2; Fig. 1).

The presence of Heterocypris reptans was restricted to samples taken from fountain basins ( $\mathrm{S} 47, \mathrm{~S} 48, \mathrm{~S} 67$,
S68). The coexistence of congeneric species was detected in two samples, S16 (Ilyocypris decipiens and I. gibba) and S58 (Heterocypris incongruens and $H$. salina). Chlamydotheca incisa was the sole species that did not belong to the fauna of the considered area.

Of particular interest is the record of Candona sp. A (Fig. 5E-H) in the temporary pool Costa di Castrignano P2 (Tabs 1, 2). This candonid ostracod, left in open nomenclature, is probably a new species, whose formal taxonomic description will be given elsewhere. However, some important morphological features are reviewed here. Candona sp. A belongs to the neglectagroup, together with C. neglecta, C. lindneri, C. meerfeldiana, C. muelleri and C. angulata (Meisch 2000). This species-group is characterised by the presence of a setal group with 4 setae on the second segment of the mandibular palp. The closest congeners of $C$. sp. A are doubtlessly $C$. neglecta and $C$. lindneri. Nevertheless, $C$. sp. A can be distinguished from both the preceding species by its unique combination of valve and soft part features. In particular, the G2 claw on the antenna of the female is slightly shorter than G1 and G3 (while it is 
distinctly reduced in size in both $C$. neglecta and $C$. lindneri), the female genital lobe has a conspicuous conical process that is directed posteriorly (in the two other species the genital lobe has no posterior process, but only a small, tit-shaped posterior lobe), and a clearly larger carapace, with a length of $1.60-1.63 \mathrm{~mm}$ in adult females $(\mathrm{n}=3)$ and $1.56-1.72 \mathrm{~mm}$ in adult males $(\mathrm{n}=2)$ (both $C$. neglecta and C. lindneri are about 1.0-1.3 mm). In addition, the M-process of the penis of $C$. sp. A is distally rounded as in $C$. lindneri, but the outer lobe is approximately triangular with rounded corners (the outline is decidedly subsquarish in C. lindneri) (Fig. 6).

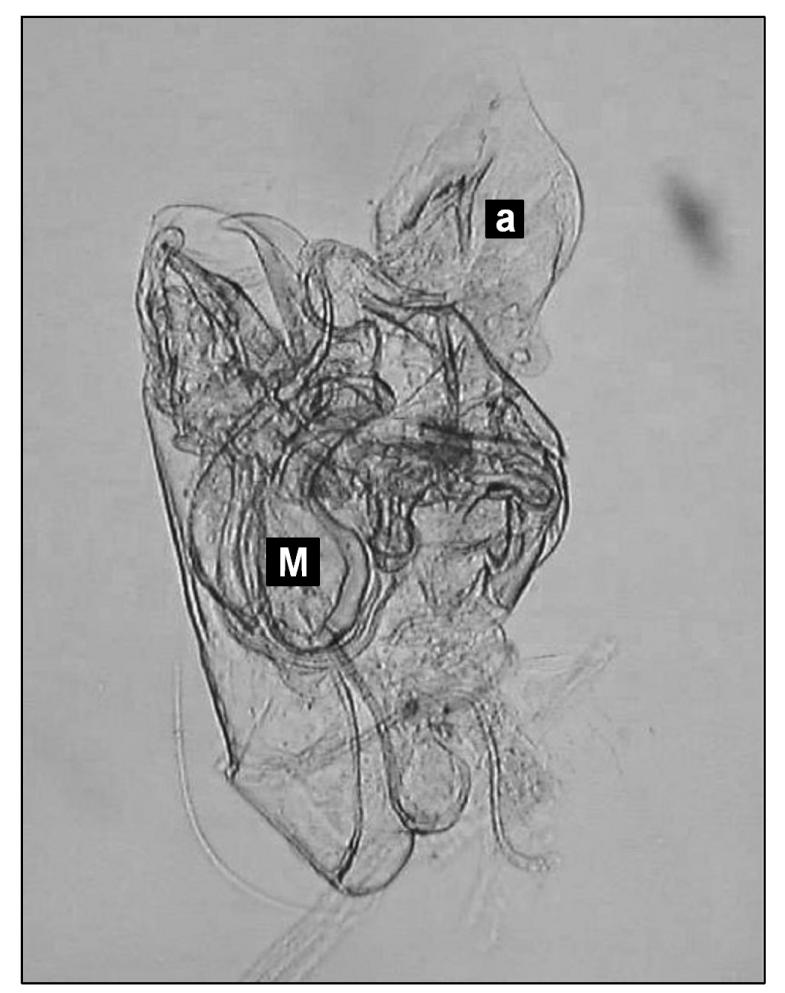

Fig. 6. Optical microscope figure of the penis of Candona sp. A (VP0619). M: M-process; a: outer lobe.

\section{DISCUSSION AND CONCLUSIONS}

This study led to the identification of an unexpectedly high number of ostracod taxa, although most of the sampled sites were visited only once, meaning that seasonal changes in the community structure were not adequately taken into account. This achievement is in part a consequence of the sampling effort which included most of the freshwater habitat types that are present in the province of Parma. Indeed, even admitting that the obtained faunal inventory is only a fraction of the taxa actually existing at the provincial scale, it still includes at least one-third of the estimated number of species present in mainland Italy and neighboring islands (Rossetti et al. 2004). The above list of ostracod taxa can be further increased by adding two species, Ilyocypris salebrosa, recorded in a study carried out in 2001 in lowland springs of the province of Parma (Rossetti et al. 2005). The presence of I. salebrosa (Fig. 7), erroneously identified as $I$. decipiens by Rossetti et al. (loc. cit., Figs $4 \mathrm{~K}, \mathrm{~L})$ and assigned here to its correct taxonomic status based on a personal communication with Dr David J. Horne (November 21, 2005), is of great interest. In fact, this species was widespread in the Holarctic Region during the Pleistocene, but recent representatives were assumed to be restricted to relict populations in Canada and China (Matzke-Karasz et al. 2004). However, other studies have shown that living populations of $I$. salebrosa exist in England (Bates et al. 2002) and Turkey (Özuluğ 2005).

Other species recorded in this study raise some interest, due to their little known occurrence in Italy. That is the case for Ilyocypris monstrifica, Potamocypris villosa, Psychrodromus olivaceus, Ps. fontinalis, Candona cf. lindneri and Physocypria kraepelini. Such a result could be due to a reduced number of investigations conducted on Recent freshwater ostracods. For example, $P$. kraepelini was repeatedly found in different habitats during recent surveys carried out in northern Italy (Pieri V., unpublished data).

The identified taxa overwhelmingly belong to the European fauna of temperate latitudes (Meisch 2000). The lone exception is Chlamydotheca incisa, a species originating from South America (Martens \& Behen 1994), that was found in two samples (S58 and S59). It is, however, a species already established in northern Italy, having been reported in ricefields (Rossi et al. 2003) and, more recently, in a lowland spring (Pieri et al. 2006b). In the checklist of the Italian non-marine ostracods prepared by Ghetti \& McKenzie (1981), the "ospiti esteri", i.e. exotic species passively introduced by man (McKenzie \& Moroni 1986), roughly accounted for $10 \%$ of the total ostracofauna. The proportion of allochtonous species found in this study is thus sensibly lower.

With few exceptions, the ostracod assemblages found in the investigated aquatic environments were characterised by a limited number of species. These observations are in accordance with data obtained in other studies which have analysed the ostracod communities of small freshwater ecosystems in Italy (Crema et al. 1996; Rossi et al. 2003; Rossetti et al. 2004, 2005; Meisch 2006; Pieri et al. 2006a, b). Moreover, no consistent differences in species richness were observed between natural and man-made ecosystems, or between sites located in protected or highly impacted areas.

As might be expected in marginal water bodies, this faunal survey confirmed a high prevalence of tolerant species able to withstand varied environmental conditions and, as a consequence, no general distributional patterns can be recognized. In only a few cases, the ostracod occurrence was clearly dependent upon the characteristics of the sampled stations (e.g., altitude, habitat persistence, and water turbulence). 


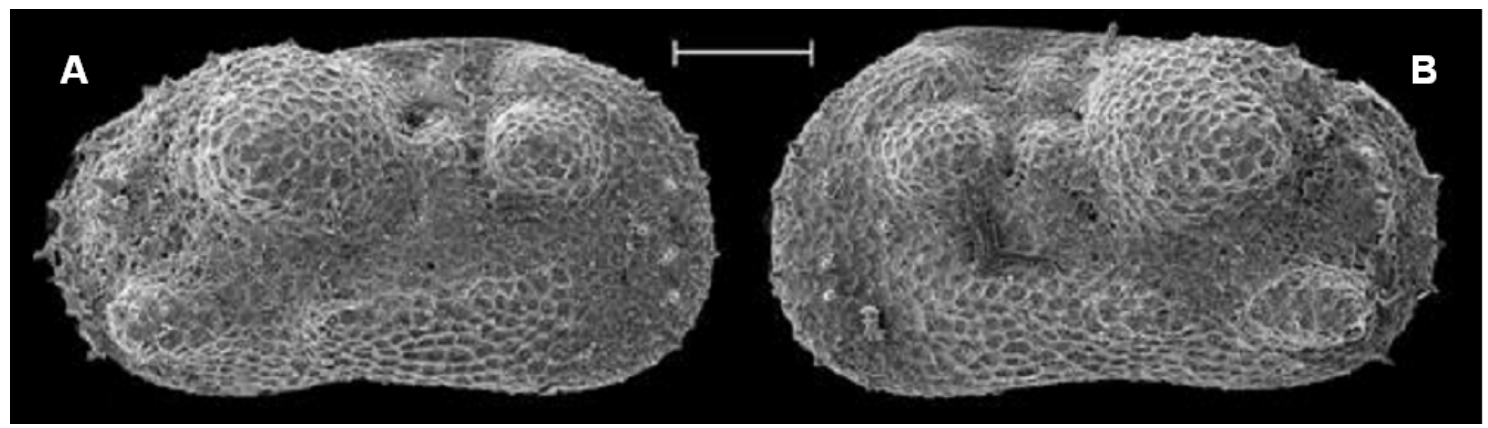

Fig. 7. Ilyocypris salebrosa (errouneously identified as I. decipiens by Rossetti et al. 2005; see text). (A): OC2824, adult female, RV, ev. (B): idem, LV, ev. Scale bar: $200 \mu \mathrm{m}$.

In conclusion, the most remarkable aspect of this study lies in the faunal analysis of aquatic bodies which are hardly considered in traditional limnological works. Indeed, minor freshwater ecosystems may represent, when considered in their whole extent, vast reservoirs of biological diversity, and in this regard they appear to be as important as larger environments.

\section{ACKNOWLEDGEMENTS}

We would like to thank the following people who assisted in different stages of this study: Antonia Cavalieri and Francesca Tireni (Parco Regionale Boschi di Carrega), Michele Adorni and Andrea Saccani (Riserva Naturale Monte Prinzera), Franca Zanichelli (Parco Regionale Fluviale del Taro), Ireneo Ferrari, Roberto Antonietti, Cinzia Marchiani, Davide Dallatomasina, Ambra De Lorentiis, Chiara Spotorno, Matteo Mazzini, Olivier Schmit, Simone Guareschi, and Giorgio Benassi (University of Parma), Ricardo L. Pinto (University of São Paulo). We are grateful to David J. Horne (Queen Mary, University of London) for establishing the correct taxonomic placement of Ilyocypris salebrosa and for providing information on its geographic distribution. Julien Cilis (R.B.I.N.Sc., Brussels) offered technical assistance with the scanning electron micrographs. An anonymous reviewer is thanked for helpful comments on the manuscript. Ostracods were partly identified during a visit of VP to KM founded by the EU Project SYNTHESYS. GR, KM and CM acknowledge the support of the EU Project SEXASEX (MRTN-CT-2004512492).

\section{REFERENCES}

Bates, M.R., D.H. Keen, J.E. Whittaker, J.S. Merry \& F.F. Wenban-Smith. 2002. Middle Pleistocene molluscan and ostracod faunas from Allhallows, Kent, UK. Proc. Geologists' Association, 113: 223-236.

Crema, S., U. Ferrarese, D. Golo, P. Modena, B. Sambugar \& R. Gerecke. 1996. Ricerche sulla fauna bentonica ed interstiziale di ambienti sorgentizi in area alpina e prealpina. Report Centro Ecologia Alpina, 8: 1-106.

Fox, H.M. 1965. Ostracod Crustacea from ricefields in Italy. Mem. Ist. ital. Idrobiol., 18: 205-214.
Ghetti, P.F. \& K. McKenzie. 1981. Ostracodi (Crustacea: Ostracoda). Guide per il riconoscimento delle specie animali delle acque interne italiane. Consiglio Nazionale delle Ricerche: $83 \mathrm{pp}$.

González Mozo, M.E., K. Martens \& A. Baltanás. 1996. A taxonomic revision of European Herpetocypris Brady and Norman, 1889 (Crustacea, Ostracoda). Bull. Inst. R. Sci. Nat. Belg., Biologie, 66: 93-132.

Martens, K. \& F. Behen. 1994. A checklist of the non-marine ostracods (Crustacea, Ostracoda) from South-American inland waters and adjacent islands Trav. scient. Mus. nat. Hist. nat. Luxembourg, 22: $81 \mathrm{pp}$.

Matzke-Karasz, R., R.J. Smith \& M. Homma. 2004. Cyclocypris diebeli Absolon, 1973 (Ostracoda, Crustacea), extinct in Europe, extant in Japan. J. Nat. Hist., 38: 16351663.

McKenzie, K.G. \& A. Moroni. 1986. Man as an agent of crustacean passive dispersal via useful plants - exemplified by Ostracoda ospiti esteri of the Italian ricefields ecosystem - and implications arising therefrom. J. Crust. Biol., 6: 181-198.

Meisch, C. 2000. Freshwater Ostracoda of Western and Central Europe. Spektrum Academischer Verlag GmbH Heidelberg, Berlin: 522 pp.

Meisch, C. 2006. Ostracoda (Muschelkrebse). In: B. Sambugar, G. Dessi, A. Sapelza, A. Stenico, B. Thaler \& A. Veneri. Südtiroler Quellfauna. Biologisches Landeslabor, Autonome Provinz Bozen, Südtirol, Italien: 315-317.

Özuluğ, O. 2005. Living specimens of Ilyocypris salebrosa Stepanaitys, 1959 (Crustacea: Ostracoda) from Thrace, Turkey. Zool. Middle East, 34: 114-116.

Pieri, V., K. Martens, L. Naselli-Flores, F. Marrone \& G. Rossetti. 2006a. Distribution of Recent ostracods in inland waters of Sicily (Southern Italy). J. Limnol., 65(1): 1-8.

Pieri, V., C. Caserini, S. Gomarasca, K. Martens \& G. Rossetti. (2006b). Water quality and diversity of the Recent ostracod fauna in lowland springs from Lombardy (northern Italy). Hydrobiologia: (in press).

Rossetti, G., M. Bartoli \& K. Martens. 2004. Limnological charateristics and recent ostracods (Crustacea, Ostracoda) of freshwater wetlands in the Parco Oglio Sud (Northern Italy). Ann. Limnol., 40: 329-341.

Rossetti, G., V. Pieri \& K. Martens. 2005. Recent ostracods (Crustacea, Ostracoda) found in lowland springs of the provinces of Piacenza and Parma (Northern Italy). Hydrobiologia, 542: 287-296.

Rossi, V., G. Benassi, M. Veneri, C. Bellavere, P. Menozzi, A. Moroni \& K.G. McKenzie. 2003. Ostracoda of the Italian ricefield thirty years on: new synthesis and hypothesis. $J$. Limnol., 62: 1-8. 